\title{
EJERCICIO AERÓBICO Y DE FUERZA EN PERSONAS CON UNA ENFERMEDAD PULMONAR OBSTRUCTIVA (EPOC): ESTUDIO DE CASO
}

\section{AEROBIC AND STRENGTH EXERCISES IN PATIENTS WITH CHRONIC OBSTRUCTIVE PULMONARY DISEASE (COPD): A CASE STUDY}

\author{
Lara Blas ${ }^{11}$ \\ Daniel Castillo ${ }^{l}$ \\ Oscar Lacalzada \\ Aitor Iturricastillo ${ }^{1}$ \\ Facultad de Educación y Deporte, Universidad del País Vasco, UPV/EHU ${ }^{1}$ \\ Club Deportivo Zuzenak², \\ Vitoria-Gasteiz, España \\ aitor.iturricastillo@ehu.eus
}

\section{Resumen}

El objetivo del estudio fue describir las respuestas fisiológicas de pacientes con EPOC en cada una de las sesiones de un programa de entrenamiento físico de ocho semanas y analizar los efectos producidos por el programa en el rendimiento físico de estos pacientes. En este estudio participaron cuatro personas a las que se les diagnosticó EPOC (64 6 años). Se realizó un test (T1) de 6 minutos de caminata (6MWT) para determinar la capacidad cardiovascular de los participantes y, después de ocho semanas, se volvió a repetir el mismo test (T2). Durante las ocho semanas se llevó a cabo trabajo de resistencia aeróbica e interválica, de fuerza, estiramientos y trabajo de musculatura respiratoria. No se observó un aumento significativo en las variables fisiológicas (tensión sistólica, tensión diastólica y saturación de oxígeno) tras la realización del 6MWT ni en el T1 ni en el T2. Sin embargo, la tensión sistólica, tanto en el Post test del T1 $(\Delta \%=24.8 \pm 22.1 ; \mathrm{TE}=2.2$, alto $)$ como en el Post test del T2 $(\Delta \%=14.0 \pm 13.2 ; \mathrm{TE}=1.0$, alto), presentó una tendencia a ser superior con respecto al Pre test, en ambos casos. Por otro lado, los participantes recorrieron ligeramente más metros después de 8 semanas de intervención $(\Delta \%=4,61 ; \mathrm{TE}=0.4$, bajo), lo cual se acompañó, de una mayor percepción del esfuerzo $(\Delta \%=107.1 ; \mathrm{TE}=3.0 \mathrm{y}$ $\Delta \%=200.0 ; \mathrm{TE}=1.8$ para RPEmus). Los participantes en este estudio obtuvieron una ligera mejora en el rendimiento físico en el test 6MWT, posiblemente debido a que las sesiones no se enfocaron solamente en la mejora de la capacidad aeróbica, sino también en la mejora de la fuerza muscular.

Palabras clave: actividad física, respuestas fisiológicas, carga interna, capacidad física.

\begin{abstract}
This study was aimed at describing the physiological responses of Chronic Obstructive Pulmonary Disease (COPD) patients in each session of an 8-week training program and analyzing its effects on the patients' physical performance. Four COPD patients ( $64 \pm 6$ years) participated in this study. A 6-minute walking test (6MWT) (T1) was performed before the training program to determine the cardiovascular capacity of the participants. After 8 weeks 6MWT was repeated (T2). During the training program participants performed interval aerobic endurance, strength, stretching and respiratory muscle training. There was no significant increase in the physiological variables (systolic and diastolic blood pressure and oxygen saturation) after
\end{abstract}


6MWT neither in T1 or T2. However, the systolic pressure in the Post test of T1 $(\Delta \%=24.8 \pm 22.1 ; \mathrm{ES}=2.2$, high $)$ and Post test of T2 $(\Delta \%=14.0 \pm 13.2 ; \mathrm{ES}=1.0$, high $)$ reported a tendency to be higher than the Pre test. With respect to physical performance, participants walked slightly more meters after the training sessions $(\Delta \%=4.61 ; \mathrm{ES}=0.4$, low $)$, which was accompanied by an increased effort perception $(\Delta \%=107.1 ; \mathrm{ES}=3.0$ and $\Delta \%=200.0 ; \mathrm{ES}=1.8$ for RPEmus). Participants slightly improved their physical performance in the $6 \mathrm{MWT}$ probably because sessions were not only focused on improving aerobic capacity but also muscle strength.

Keywords: physical activity, physiological responses, internal load, physical capacity.

\section{Introducción}

La enfermedad pulmonar obstructiva crónica (EPOC) es un estado patológico no totalmente reversible caracterizado por una limitación del flujo de aire que ingresa a los pulmones (Global Initiative for Chronic Obstructive Lung Disease [GOLD], 2016). Esta limitación es habitualmente progresiva y está asociada con una respuesta inflamatoria anormal de los pulmones frente a partículas nocivas o a gases. Aunque el tabaco sea la causa principal y la más estudiada, el fumado no es el único factor de riesgo de la EPOC. Existen otros factores, tales como: los genéticos; la edad y el género; el crecimiento y el desarrollo de los pulmones; las infecciones respiratorias en la infancia; el nivel socioeconómico; el asma y la hiperreactividad bronquial; la bronquitis crónica Estos pueden ser los causantes de dicha enfermedad (GarcíaAymerich, 2005; Menezes y Knorst, 2005; Montes, 2011).

Para combatir esta afección, actualmente el tratamiento farmacológico es imprescindible (Bardagi y Solans, 2007; Viejo, 2005). Sin embargo, existen otras medidas que pueden mejorar la calidad de vida de las personas que padecen esta enfermad, por ejemplo, mediante el uso de técnicas específicas como la oxigenoterapia (Escarrabil, 2005; Fernández-Fernández et al., 2003), la ventilación no invasiva (GOLD, 2016) o trabajos quirúrgicos (GOLD, 2016). Además, investigaciones recientes han demostrado que la realización de actividad física es uno de los pilares básicos en la rehabilitación pulmonar (Covey et al., 2012; Iepsen et al., 2015; Rocha Leite et al., 2015) debido a que se ha reconocido que los programas de actividad física de mínimo ocho semanas son un tratamiento efectivo (Troosters, Gosselink, Janssens y Decramer, 2010).

Los pacientes con EPOC reducen su nivel de actividad debido a la sensación de disnea, lo cual favorece que adopten un estilo de vida sedentario: se disminuye progresivamente el volumen de ejercicio físico. Lo anterior genera un círculo vicioso de inactividad y pérdida de las capacidades físicas (British Thoracic Society Standards of Care Subcommittee on Pulmonary Rehabilitation, 2001; Fierro-Carrión y Mahler, 2002; Vargas, 2003). Este hecho favorece que la disnea progrese, hecho que origina un descenso en la capacidad aeróbica y una disminución de la masa corporal; consecuentemente, también se produce un descenso de la fuerza muscular (British Thoracic Society Standards of Care Subcommittee on Pulmonary Rehabilitation, 2001; Fierro-Carrión y Mahler, 2002; Vargas, 2003). 
Sin embargo, el ejercicio aeróbico les ofrece beneficios a los pacientes con EPOC, ya que a través de una mejora de la capacidad aeróbica y funcional, lograda mediante ejercicio de alta intensidad (Troosters, Gosselink y Decramer, 2000) y ejercicio interválico y continuo (Puhan et al., 2006), se pueden reducir síntomas como la disnea, la intolerancia al ejercicio o la reducción de la calidad de vida (Andrianopoulos, Klijn, Franssen y Spruit, 2014; Rocha Leite et al., 2015; Wadell et al., 2013). La capacidad aeróbica y funcional se ha medido por medio del test de caminata de 6 minutos (6MWT) por varios autores (Albarrati et al., 2016; Saglam et al., 2015); no obstante, son pocos los estudios que han utilizado esta herramienta para valorar la mejora antes y después de un programa de intervención.

Por otro lado, el entrenamiento de fuerza muscular aumenta la fuerza y la masa de la musculatura ejercitada y, junto con el entrenamiento aeróbico, consigue incrementos adicionales de la fuerza de la musculatura periférica (Bolton et al., 2013; Güell et al., 2014; Spruit et al., 2013). Además de la mejora muscular, este tipo de entrenamiento mantiene o, en algunos casos, incluso incrementa la densidad mineral ósea (Bolton et al., 2013; Güell et al., 2014; Spruit et al., 2013). Según Iepsen et al. (2015), el entrenamiento de fuerza mejora la capacidad de realizar ejercicio, la calidad de vida (Lacasse et al., 2006) y reduce el número de días de ingreso en el hospital (Griffiths et al., 2000). El fortalecimiento de las extremidades superiores podría reducir la disnea y mejorar la capacidad aeróbica, así como aumentar la fuerza de la pared torácica y de los músculos respiratorios (Covey et al., 2012; O’Shea, Taylor y Paratz, 2007). En esta línea, el trabajo de la musculatura inspiratoria adquiere tanta importancia como el fortalecimiento de los músculos de las extremidades superiores (Bernardi, Pomidori, Bassal, Contoli y Cogo, 2015).

Para trabajar cuidadosamente la actividad física en personas con EPOC, varias investigaciones han sido llevadas a cabo. Estas investigaciones han combinado programas mixtos de entrenamiento: ejercicio aeróbico, de fuerza muscular y de músculos inspiratorios. Sin embargo, la mayoría de estos estudios sobrepasa las 8 semanas de duración (Ortega et al., 2002; Troosters et al., 2000; Vonbank et al., 2012) y es escasa la literatura que determina las respuestas fisiológicas de cada sesión atendiendo a la tensión arterial y a la saturación de oxígeno (Martin et al., 1992).

En vista de lo anterior, el primer objetivo del estudio fue establecer o analizar los efectos de un programa de entrenamiento mixto (capacidad aeróbica, fuerza, estiramientos, musculatura respiratoria) de ocho semanas mediante el test de 6 minutos de caminata (6MWT), en pacientes con EPOC. Por otro lado, el segundo objetivo fue describir y analizar las respuestas fisiológicas de los pacientes con EPOC en cada una de las sesiones.

\section{Método}

\section{Participantes}

Los participantes del estudio fueron tres hombres y una mujer diagnosticados de EPOC $\left(64 \pm 6\right.$ años, $\left.84.5 \pm 20.1 \mathrm{~kg}, 168.0 \pm 13.6 \mathrm{~cm}, 29.5 \pm 2.6 \mathrm{~kg} \cdot \mathrm{m}^{-2}\right)$, los cuales asisten a un centro 
de actividad física adaptada (Tabla 1). Se utilizó un total de 76 registros para cada variable. A todos los participantes se les explicaron los riesgos y beneficios de la participación en el estudio y firmaron el preceptivo consentimiento informado; además, podían retirarse del estudio en cualquier momento. La investigación siguió las pautas marcadas en la Declaración de Helsinki (2013) y se realizó con base en estándares éticos establecidos para investigaciones en ciencias del deporte y del ejercicio (Harris y Atkinson, 2013).

Tabla 1. Descripción de los valores antropométricos de los participantes

\begin{tabular}{cccccc}
\hline Participantes & 1 & 2 & 3 & 4 & Total \\
\hline Grado de EPOC & Severo & Moderado & Severo & Moderado & - \\
Edad (años) & 64 & 60 & 60 & 73 & $64 \pm 6$ \\
Peso (kg) & 59 & 108 & 84 & 87 & $84.5 \pm 20.1$ \\
Altura (cm) & 150 & 183 & 171 & 168 & $168.0 \pm 13.6$ \\
IMC & 26.2 & 32.3 & 28.7 & 30.8 & $29.5 \pm 2.6$ \\
FVC (\%) & 60 & - & 76 & 104 & $80.0 \pm 22.3$ \\
FEV $_{1}(\%)$ & 54 & - & 49 & 42 & $48.3 \pm 6.0$ \\
\hline
\end{tabular}

$E P O C=$ enfermedad pulmonar obstructiva crónica; $I M C=$ índice de masa corporal; $F V C=$ capacidad vital forzada; $F E V_{1}=$ volumen espiratorio forzado en 1 segundo.

\section{Procedimiento}

En el mes de enero (2016) se llevó a cabo una sesión de trabajo para valorar el rendimiento físico de los participantes (T1) mediante el test de 6 minutos de caminata (6MWT). Al final de ocho semanas de intervención, en marzo, se volvió a repetir el mismo test (T2) - los participantes fueron instruidos para realizar el 6MWT a la máxima intensidad posible-. Antes de la realización de los test se realizó un calentamiento estándar que consistió en 10 minutos de ejercicio aeróbico en la cinta o la elíptica según las necesidades de cada participante. Después del calentamiento los participantes reposaron mientras que se les explicaba cómo realizar el test. Las sesiones de test se realizaron en las horas correspondientes a las sesiones dirigidas establecidas por el centro de actividad física adaptada, siempre en horas de la mañana (entre las 11:00 a.m. y las12:00 p.m.), en un gimnasio.

\section{Test de 6 minutos de caminata (6MWT)}

El 6MWT fue realizado por todos los participantes el mismo día. Los participantes fueron instruidos para caminar durante 6 minutos a lo largo de un pasillo llano de 25 metros, esto con la intención de cubrir la máxima distancia posible. Ningún participante repitió más de una vez 
el test $\mathrm{y}$, durante este, los investigadores motivaron y animaron a los participantes para que realizaran la prueba con la mayor intensidad posible. La distancia recorrida (m) por cada uno de los participantes se utilizó para realizar el análisis estadístico.

\section{Mediciones}

Se llevaron a cabo las siguientes mediciones.

Tensión arterial. La tensión arterial, tanto sistólica ( $\mathrm{mmHg}$ ) como diastólica ( $\mathrm{mmHg}$ ), fue tomada con el monitor de tensión arterial (Omron M3 Intellisense, Alemania). Antes (Pre) y después (Post) del 6MWT, así como antes y después de todas las sesiones, se determinó la tensión arterial de los participantes. Tanto el valor de la tensión sistólica (TS) como el de la diastólica (TD) fueron utilizados para el análisis estadístico.

Saturación de oxígeno. El porcentaje (\%) de la saturación de oxígeno se determinó mediante el pulsioxímetro (Konika Minolta Pulsox-300i, United States), antes y después de haber terminado las sesiones y los test de 6MWT. La saturación de oxígeno se midió en el dedo anular de la mano izquierda en todos los casos. Ese valor único fue utilizado para el análisis estadístico.

Esfuerzo percibido. La escala de 0-10 puntos propuesta por Foster y col. (2001) fue empleada para obtener la percepción subjetiva del esfuerzo realizado. Los participantes respondieron, al final de cada $6 \mathrm{MWT}$ y de cada sesión, acerca de la percepción del esfuerzo de forma diferenciada (dRPE): desde los puntos de vista respiratorio (RPEres) y muscular (RPEmus). Posteriormente, los valores de RPEres y RPEmus de las sesiones se multiplicaron por la duración total de estas (min), de acuerdo con Foster et al. (2011), para obtener la carga interna derivada del RPE, tanto respiratorio (sRPEres) como muscular (sRPEmus), en unidades arbitrarias (UA). Los participantes fueron familiarizados con el uso de esta escala en las dos semanas previas a la intervención.

\section{Programa de intervención}

El programa de intervención duró ocho semanas, en las cuales se realizaron tres sesiones semanales de 60 minutos, separadas entre sí por 48 horas de descanso. Cada una de las sesiones se estructuró de la siguiente forma. En primer lugar, se realizó un calentamiento de 15-20 minutos, ya fuera sobre el tapiz rodante o la bicicleta estática y/o elíptica, de manera continua y a baja intensidad. En segundo lugar, se pasó a una parte principal de 35-40 minutos cuyo objetivo se dirigió a la resistencia aeróbica interválica, a la potenciación de las extremidades superiores e inferiores, al trabajo de core y a la musculatura inspiratoria. En tercer lugar, se llevó a cabo estiramientos generales y ejercicios de relajación, los cuales tenían una duración de 5-7 minutos.

El ejercicio interválico se basó en combinar periodos cortos de alta intensidad, al 70 \%-75\% de la frecuencia cardíaca máxima, con periodos de menor intensidad de igual o menor duración. La intensidad se individualizó mediante la fórmula de Karvonen (Karvonen y 
Vuorimaa, 1988). El ejercicio se realizó sobre bicicleta estática o elíptica, tapiz rodante y step, de forma alternada o en cancha combinando diferentes ejercicios. La carga fue aumentando semanalmente de forma graduada, a través del incremento de la duración de la repetición en alta intensidad e incorporando nuevas repeticiones y series. En cuanto a la velocidad, esta fue controlada por los participantes y se centró en el cambio de ritmo.

El programa de fuerza se completó mediante ejercicios dirigidos a potenciar las extremidades superiores e inferiores, con diverso material: se realizó un circuito de 8-10 estaciones o de forma guiada. Además, se potenció el core a través de ejercicios específicos para esta musculatura y con control de la respiración. La intensidad fue aumentando semanalmente, incrementando la duración del tiempo de trabajo en cada estación en el caso del circuito y añadiendo nuevas repeticiones en el caso del trabajo guiado y del core.

El entrenamiento de la musculatura inspiratoria se ejecutó a través de cinco ejercicios, de forma guiada, con diferente material, controlando la respiración y de manera autónoma, con el dispositivo de carga resistiva Power-Breathe. Se realizaron diariamente 2 series de 30 respiraciones con la mayor carga de dificultad posible, de forma continua o de forma fraccionada hasta alcanzar el total, en el caso de no poder completar las series de forma continua.

Durante este periodo se cuantificaron los cambios en la tensión arterial y la saturación de oxígeno en todas las sesiones (5 minutos antes e inmediatamente después de terminar la sesión), así como la carga interna subjetiva (10 minutos después de terminar la sesión). La carga interna subjetiva obtenida en el estudio se presenta en la figura 1.

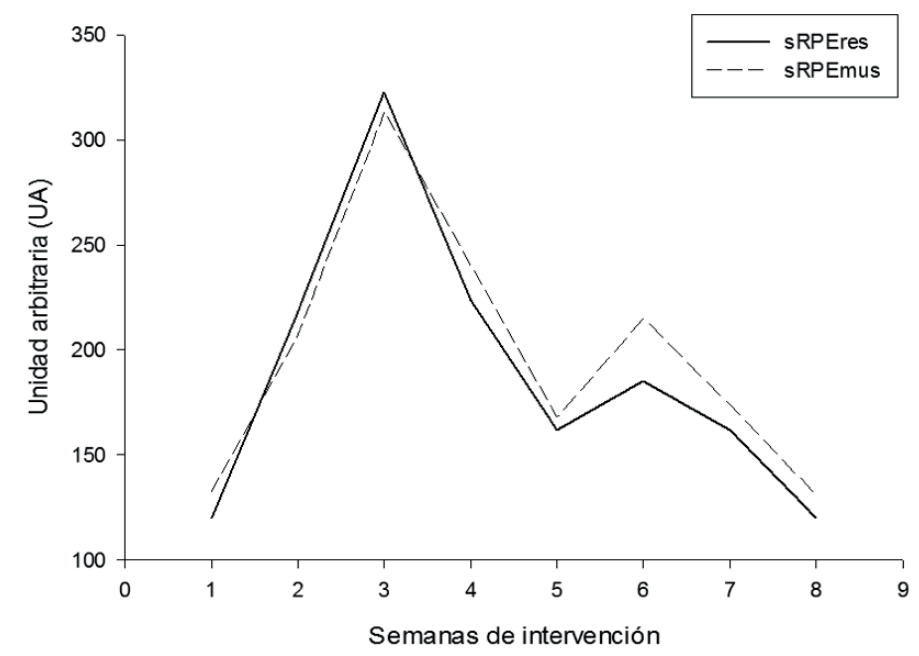

Figura 1. Carga interna semanal medida mediante métodos subjetivos diferenciados (sRPEres y sRPEmus). 


\section{Análisis estadístico}

Los resultados se presentan como media \pm desviación típica (DT) de la media. La normalidad de los datos se analizó mediante las pruebas de Kolmogorov-Smirnov y Saphiro-Wilks, con el fin de verificar la necesidad de pruebas paramétricas o no paramétricas. En este sentido, se utilizaron las pruebas no paramétricas. Se utilizó la prueba no paramétrica de Wilcoxon para determinar las diferencias entre el Pre y Post semanal (tensión sistólica, tensión diastólica y saturación de oxígeno). Además, también sirvió para determinar las diferencias entre el Pre y Post (T1-T2) programa de intervención en las respuestas fisiológicas, la distancia recorrida y el esfuerzo percibido diferenciado. Para determinar la magnitud del cambio se calculó el porcentaje del cambio $\Delta \%=[($ Post-Pre $) /$ Pre $]$ x100. Además, se calculó el tamaño del efecto (TE), con base en el método propuesto por Cohen (1988) (Media Post - Media Pre)/DT Post. Tamaños del efecto menores a 0.2, entre 0.2-0.5, entre 0.50.8 o mayores de 0.8 fueron considerados trivial, bajo, moderado o alto, respectivamente. El análisis estadístico se realizó con el programa Statistical Package for Social Sciences (SPSS Inc, versión 20,0 Chicago, IL, EE.UU.). El nivel de significación estadística fue establecido en $\mathrm{P}<0,05$.

\section{Resultados}

No se observaron diferencias significativas en relación con las respuestas fisiológicas (tensión arterial y saturación de oxígeno) entre los test Pre y el Post sesión durante todo el programa de intervención (Tabla 2). Sin embargo, en la semanas 5 (de $15.4 \pm 1.7$ a $14.5 \pm 1.6 \mathrm{mmHg}$ ), 6 (de $14.9 \pm$ 1.4 a $14.1 \pm 1.6 \mathrm{mmHg}$ ) y 7 (de $15.2 \pm 2.0$ a $14.0 \pm 1.8$ ) la TS fue menor en el test Post sesión que en el Pre sesión (rango TE $=-0.53$ a -0.60 , moderado). La TD fue mayor en la semana 1 (de $7.4 \pm 1.4$ a $8.1 \pm 1.2 ; \mathrm{TE}=0.5$, moderado) y menor en las semana 7 ( $\mathrm{TE}=0.5$, moderado). La saturación de oxígeno, por su parte, fue más baja en la semana 2 (de $95.5 \pm 1.6$ a $94.2 \pm 1.3$; $\mathrm{TE}=0.8$, moderado).

Tabla 2. Descripción semanal de los resultados obtenidos en relación con la tensión arterial y la saturación de oxígeno antes (Pre) y después (Post) de las sesiones en sujetos con EPOC

\begin{tabular}{|c|c|c|c|c|c|c|c|c|c|c|c|c|}
\hline & \multicolumn{4}{|c|}{ Tensión sistólica (mmHg) } & \multicolumn{4}{|c|}{ Tensión diastólica (mmHg) } & \multicolumn{4}{|c|}{ Saturación de oxigeno (\%) } \\
\hline & Pre & Post & $\Delta \%$ & $\mathrm{TE}$ & Pre & Post & $\Delta \%$ & $\mathrm{TE}$ & Pre & Post & $\Delta \%$ & TE \\
\hline S1 & $14.7 \pm 1.9$ & $14.9 \pm 1.9$ & 1.4 & 0.1 & $7.4 \pm 1.4$ & $8.1 \pm 1.2$ & 9.5 & 0.5 & $94.8 \pm 1.6$ & $94.9 \pm 1.3$ & 0.1 & 0.1 \\
\hline $\mathrm{S} 2$ & $15.2 \pm 1.8$ & $14.4 \pm 1.8$ & -5.3 & -0.4 & $7.8 \pm 1.2$ & $7.7 \pm 1.1$ & -1.3 & -0.1 & $95.5 \pm 1.6$ & $94.2 \pm 1.3$ & -1.4 & -0.8 \\
\hline S3 & $15.7 \pm 2.3$ & $14.7 \pm 2.3$ & -6.4 & -0.4 & $7.3 \pm 1.3$ & $7.2 \pm 1.3$ & -1.4 & -0.1 & $95.6 \pm 0.8$ & $95.4 \pm 1.1$ & -0.2 & -0.3 \\
\hline S4 & $14.7 \pm 2.6$ & $14.0 \pm 1.1$ & -4.8 & -0.3 & $7.1 \pm 1.3$ & $7.6 \pm 1.1$ & 7.0 & 0.4 & $94.5 \pm 1.4$ & $94.5 \pm 1.1$ & 0.0 & 0.0 \\
\hline S5 & $15.4 \pm 1.7$ & $14.5 \pm 1.6$ & -5.8 & -0.5 & $7.0 \pm 1.1$ & $7.4 \pm 0.8$ & 5.7 & 0.4 & $95.0 \pm 1.2$ & $94.9 \pm 1.3$ & -0.1 & -0.1 \\
\hline S6 & $14.9 \pm 1.4$ & $14.1 \pm 1.6$ & -5.4 & -0.6 & $7.5 \pm 1.2$ & $7.4 \pm 1.4$ & -1.3 & -0.1 & $94.8 \pm 1.4$ & $94.9 \pm 1.5$ & 0.1 & 0.1 \\
\hline S7 & $15.2 \pm 2.0$ & $14.0 \pm 1.8$ & -7.9 & -0.6 & $8.1 \pm 1.2$ & $7.5 \pm 1.3$ & -7.4 & -0.5 & $95.4 \pm 2.0$ & $94.6 \pm 1.2$ & -0.9 & -0.5 \\
\hline S8 & $13.9 \pm 1.5$ & $14.0 \pm 1.8$ & 0.7 & 0.1 & $7.5 \pm 1.0$ & $7.8 \pm 1.3$ & 4.0 & 0.3 & $94.6 \pm 1.7$ & $94.7 \pm 1.2$ & 0.1 & 0.1 \\
\hline
\end{tabular}

Los resultados son medias $\pm D T ;$ EPOC = enfermedad pulmonar obstructiva crónica. TE = tamaño del efecto, $S=$ semana; $P>0.05$, no existen diferencias significativas en ningún caso. 
Por otro lado, los resultados en el 6MWT, tanto en el Pre como en el Post test, en relación con las respuestas fisiológicas, se muestran en la Tabla 3. No se observaron diferencias significativas $(p<0.05)$, en ninguna de las variables, ni en el T1 ni en el T2. Sin embargo, hubo una tendencia a que la TS en el Post test del T1 $(\mathrm{TS}=$ de $14.4 \pm 1.5$ a $17.7 \pm 1.9 \mathrm{mmHg} ; \Delta \%=$ $24.8 \pm 22.1 ; \mathrm{TE}=2.2$, alto) y en el Post test del T2 (TS $=$ de $14.7 \pm 1.9$ a $16.6 \pm 2.3 \mathrm{mmHg} ; \Delta \%$ $=14.0 \pm 13.2 ; \mathrm{TE}=1.0$, alto) fuera mayor que en el Pre test. Por otro lado, las diferencias fueron mínimas tanto en la TD como en la saturación de oxígeno.

Tabla 3. Respuestas fisiológicas antes (Pre) y después (Post) del test de 6 minutos de caminata (6MWT) y del programa de intervención (T1-T2)

\begin{tabular}{|c|c|c|c|c|c|c|c|c|}
\hline & \multicolumn{4}{|c|}{$\mathrm{T} 1$} & \multicolumn{4}{|c|}{$\mathrm{T} 2$} \\
\hline & Pre & Post & $\Delta \%$ & $\mathrm{TE}$ & Pre & Post & $\Delta \%$ & TE \\
\hline Tensión sistólica (mmHg) & $14.4 \pm 1.5$ & $17.7 \pm 1.9$ & $24.8 \pm 22.1$ & 2.2 & $14.7 \pm 1.9$ & $16.6 \pm 2.3$ & $14.0 \pm 13.2$ & 1.0 \\
\hline Tensión diastólica (mmHg) & $7.9 \pm 1.2$ & $7.9 \pm 1.3$ & $0.4 \pm 4.9$ & 0.0 & $7.7 \pm 1.2$ & $7.9 \pm 1.0$ & $2.9 \pm 6.3$ & 0.2 \\
\hline Saturación de oxígeno (\%) & $93.8 \pm 2.5$ & $94.3 \pm 3.5$ & $1.1 \pm 1.5$ & 0.2 & $94.5 \pm 0.6$ & $91.5 \pm 1.7$ & $-3.2 \pm 1.7$ & -5.0 \\
\hline Distancia (m) & - & $541.8 \pm 65.3$ & - & - & & $566.8 \pm 72.4$ & - & - \\
\hline RPEres (UA) & - & $2.8 \pm 1.0$ & - & - & & $5.8 \pm 1.0$ & - & - \\
\hline RPEmus (UA) & - & $2.0 \pm 2.2$ & - & - & & $6.0 \pm 2.2$ & - & - \\
\hline
\end{tabular}

Los resultados son medias $\pm D T$. TE = tamaño del efecto; RPEres = percepción del esfuerzo respiratorio; $R P E-$ mus $=$ percepción del esfuerzo muscular; $U A=$ unidad arbitraria. $P>0.05$, no existen diferencias significativas en ningún caso.

Con respecto a las diferencias entre el $\mathrm{T} 1 \mathrm{y}$ el T2, no hubo diferencias significativas en cuanto a la tensión arterial. A pesar de ello, los participantes recorrieron ligeramente más metros $(541.8 \pm 65.3$ y $566.8 \pm 72.4$ para T1 y T2, respectivamente) después de 8 semanas de intervención $(\Delta \%=4,61$; TE $=0.4$, bajo $)$, resultado que se acompañó de una mayor percepción del esfuerzo $(\Delta \%=107.1 ; \mathrm{TE}=3.0$ para RPEres y $\Delta \%=200.0 ; \mathrm{TE}=1.8$ para RPEmus) y de una mayor disminución en la saturación de oxígeno $(\Delta \%=1.1 \pm 1.5 ; \mathrm{TE}=0.2$ vs. $\Delta \%=-3.2 \pm 1.7 ; \mathrm{TE}=-5.0$, alto, en el T1 y el T2 respectivamente).

\section{Discusión}

Diferentes estudios han observado beneficios producto de la actividad física en participantes con EPOC, gracias a programas independientes. En dichos programas se han trabajado solamente la capacidad aeróbica (Rocha Leite et al., 2015) y la fuerza muscular (Covey et al., 2012). Además, estas mejoras también se han observado con programas mixtos que trabajan ambas capacidades (Ortega et al., 2002; Troosters et al., 2000; Vonbank et al., 2012). La mayoría de los estudios analizan programas de 12, 16 o 24 semanas de duración, por lo que son pocos los estudios que han analizado programas de 8 semanas (Mador et al., 2004). 
Por ello, el primer objetivo del estudio fue establecer o analizar los efectos de un programa de entrenamiento (capacidad aeróbica, fuerza, estiramientos, musculatura respiratoria) de 8 semanas en el rendimiento físico de pacientes con EPOC. El segundo objetivo fue describir y analizar las respuestas fisiológicas de los pacientes con EPOC en cada una de las sesiones. Los participantes recorrieron ligeramente más metros después de 8 semanas de intervención $(\Delta \%=$ 4,$61 ; \mathrm{TE}=0.4$, bajo); sin embargo, declararon mayor percepción del esfuerzo $(\Delta \%=107.1 ; \mathrm{TE}=$ 3.0 , alto, para RPEres y $\Delta \%=200.0 ; \mathrm{TE}=1.8$, alto, para RPEmus).

El control y la cuantificación de la carga se han utilizado en sujetos con EPOC mediante métodos objetivos (Chen et al., 2015; Rocha Leite et al., 2015) y subjetivos (Mador et al., 2004; Nakamura et al., 2008). Pese a ello, pocos estudios han determinado la carga interna idónea para lograr las consiguientes mejoras de las capacidades físicas (Mador et al. 2004; Nakamura et al., 2008). En el presente estudio, se utilizaron la tensión arterial y la saturación de oxígeno para determinar las respuestas fisiológicas luego de cada sesión. Los métodos subjetivos, por su parte, se emplearon para determinar la carga interna de la actividad física. En este sentido, tanto la TS como la TD fueron similares al inicio y final de las sesiones.

No obstante, en tres semanas la TS disminuyó Post sesión, mientras que la TD aumentó y disminuyó en dos semanas; además, la saturación de oxígeno disminuyó en una semana. Los individuos adultos que presentan un IMC alto, como en nuestro estudio, tienen respuestas más altas de la TS al Post esfuerzo (De Moraes et al., 2007; Skinner et al., 2005) y normalmente hay una disminución de la TD Post ejercicio (Chiacchio, Omar y Suau, 2010). En nuestro estudio, en cambio, la disparidad de los resultados Post sesión no presenta un verdadero aumento Post sesión en la TS ni tampoco una disminución en la TD. Los niveles de oxígeno normales de $95 \%$ a $100 \%$ permiten contar con la presión adecuada dentro del cuerpo para permitir que el oxígeno se absorba en los músculos (Mayer et al., 2010). En este sentido, la saturación de oxígeno estuvo rondando cerca de los parámetros normales, tanto en el Pre test (rango $=94.6$ a 95.6 \%) como en el Post test $($ rango $=94.2$ a $95.4 \%$ ), siempre teniendo en cuenta que los participantes presentaban una EPOC.

Sin embargo, mientras que los cambios en las respuestas fisiológicas no presentaban una gran variación, la carga interna subjetiva fue disminuyendo según avanzaba el programa con respecto a la semana: 2, 3 y 4 (rango $\Delta \%=-15.2$ a $-62.8 \%$ ). Otros investigadores (Mador et al., 2004) utilizaban el RPE como método para controlar la intensidad subjetiva de la actividad física y no dejaban a los participantes superar el valor 5 de la escala CR10, por lo que es difícil comparar los valores de nuestro estudio con otros. Además de describir las respuestas fisiológicas al ejercicio y cuantificar la carga interna mediante métodos subjetivos, sería interesante en futuros estudios aumentar la información con la carga interna y externa, medidas mediante métodos objetivos.

El rendimiento físico se ha medido en sujetos con EPOC con diferentes test de campo (Mador et al., 2004; Panton et al., 2004). Algunos autores (Rocha Leite et al., 2015) observan que existen mejoras en el rendimiento físico de los test de campo con un programa exclusivo de

Revista MHSalud® (ISSN: 1659-097X) Vol. 13. No. 2. Febrero-Agosto, 2017 
trabajo aeróbico. De igual manera ocurre en nuestro estudio: se reportan mejoras después de 24 sesiones de entrenamiento mixto (541.8 $\pm 65.3 \mathrm{~m}$ a $566.8 \pm 72.4 \mathrm{~m}$ recorridos), aunque son más pequeñas $(\mathrm{TE}=0.4$, bajo) que en los estudios mencionados. Mador et al. (2004) también aportaron rendimientos similares, atendiendo al aumento de la distancia recorrida en el 6MWT, y exponen mejoras no mayores a $54 \mathrm{~m}$ de media. Troosters et al. (2000) observan una mejora de $52 \mathrm{~m}$ después de una intervención mixta (ciclismo indoor, caminatas y trabajo de fuerza) de 24 semanas.

Estas diferencias en las mejoras encontradas pueden deberse no solo a que las sesiones de otros estudios (Güell et al., 2014; Mador et al., 2004) estaban compuestas por sesiones para mejorar la capacidad aeróbica exclusivamente, sino también a la duración de la intervención (Troosters et al. 2000), y al número, género y características de los participantes en los distintos estudios. En nuestro estudio, la pequeña mejora en el rendimiento físico fue acompañada por un incremento en la percepción del esfuerzo (TE $<3.0$, alto, RPEres y RPEmus respectivamente), tal vez debido al descenso de la saturación del oxígeno $(\mathrm{TE}=5.0$, alto) en el Post test del T2. A pesar de estos resultados, son necesarios más estudios que determinen específicamente qué tipo de programa de entrenamiento, con cuántas semanas de duración y con cuánta carga interna (objetiva y subjetiva) puede ofrecer una mayor mejoría con la máxima eficiencia a los pacientes con EPOC.

Los resultados obtenidos en el presente estudio se deben interpretar con precaución debido a algunas limitaciones del estudio. Por un lado, la muestra es pequeña y resulta difícil realizar generalizaciones. Además, a pesar de que todos los participantes tuvieran EPOC, no se han tenido en cuenta las posibles comorbilidades de estos. Por otro lado, un grupo control aportaría la exactitud de los efectos del programa de entrenamiento en el rendimiento físico, lo que sería interesante de hacer enfuturos estudios.

\section{Conclusiones}

Los pacientes con EPOC que participaron en este estudio obtuvieron una ligera mejora en el rendimiento del test $6 \mathrm{MWT}$ después de realizar un programa de entrenamiento mixto (capacidad aeróbica, fuerza, estiramientos, musculatura respiratoria) de 8 semanas de duración. Asimismo, se reportan valores normales de acuerdo con las respuestas fisiológicas (tensión sistólica, tensión diastólica y saturación de oxígeno) de los pacientes con EPOC durante el programa de intervención.

\section{Agradecimientos}

Agradecemos al C.D. Zuzenak por ofrecernos la posibilidad de realizar este estudio de intervención y, en particular, al profesional que dirige las sesiones y a los pacientes. 


\section{Referencias bibliográficas}

Albarrati, A.M., Gale, N.S., Enright, S., Munnery, M.M., Cockcroft, J.R., \& Shale, D.J. (2016). A simple and rapid test of physical performance in chronic obstructive pulmonary disease. International Journal of Chronic Obstructive Pulmonary Disease, 11, 1785-1791. Doi: 10.2147/COPD.S106151.

Andrianopoulos, V., Klijn, P., Franssen, F.M., \& Spruit, M.A. (2014). Exercise training in pulmonary rehabilitation. Clinical Chest Medicine, 35(2), 313-322. doi: 10.1016/j. ccm.2014.02.013

Bardagi, S. \& Solans, M. (2007). Enfermedad pulmonar obstructiva crónica. Prevalencia. Clínica. Tratamiento farmacológico. En E. Pleguezuelos., G. Miranda., A. Gómez \& L. Capellas (coords.), Rehabilitación integral en el paciente con enfermedad pulmonar obstructiva crónica (pp. 22-32). Madrid: Editorial Médica Panamericana.

Bernardi, E., Pomidori, L., Bassal, F., Contoli, M., \& Cogo, A. (2015). Respiratory muscle training with normocapnic hyperpnea improves ventilatory pattern and thoracoabdominal coordination, and reduces oxygen desaturation during endurance exercise testing in COPD patients. International Journal of COPD, 10, 1899-1906. doi: 10.2147/COPD.S88609

Bolton, C.H.E., Bevan-Smith, E.F., Blakey, J.D., Crowe, P., Elkin, S.L., Garrod, R., (...) \& British Thoracic Society Standards of Care Committee. (2013). British Thoracic Society guideline on pulmonary rehabilitation in adults. Thorax, 68, 1-30. doi: 10.1136/thoraxjnl-2013-203808

British Thoracic Society Standards of Care Subcommittee on Pulmonary Rehabilitation (2001). BTS Statement: Pulmonary Rehabilitation. Thorax, 56, 827-834. doi: 10.1136/ thorax.56.11.827

Chen, C-A., Chang, C-H., Lin, M-T., Hua, Y-C., Fang, W-Q., Wu, M-H., (...) \& Wang, J-K. (2015). Six-minute walking test: normal reference values for taiwanese children and adolescents. Acta Cardiologica Sinica, 31(3), 193-201. doi: 10.6515/ACS20140721D

Chiacchio, M., Omar, A., \& Suau, R. (2010).Respuesta de la tensión arterial a la prueba de esfuerzo. Apunts: Medicina de l'esport, 45(167), 191-200. doi:10.1016/j.apunts.2010.01.005

Covey, M.K., McAuley, E., Kapella, M.C., Collins, E.G., Alex, C.G., Berbaum, M.L., \& Larson, J.L. (2012). Upper-body resistance training and self-efficacy enhancement in COPD. Journal of Pulmonary and Respiratory Medicine, (supl. 9:001). doi:10.4172/2161-105X.S9-001

Cohen, J. (1988). Statistical power analysis for the behavioral sciences. New York: Academic Press. 
De Moraes Chaves Becker, M., Barbosa e Silva, O., Gonçalves Moreira, I.E., \& Guimarães Victor, E. (2007). Pressão Arterial em Adolescentes Durante Teste Ergométrico. Arquivos Brasileiros de Cardiologia, 88, 329-33. doi: 10.1590/S0066-782X2007000300012

Escarrabill, J. (2005). Indicaciones de la oxigenoterapia domiciliaria en la enfermedad pulmonar obstructiva crónica. En A. Agustí \& B. Celli (Ed.), Enfermedad pulmonar obstructiva crónica (pp. 221-228). Bercelona, España: Masson.

Fierro-Carrion, G., \& Mahler, D.A. (2002). Exercise prescription for patients with chronic lung disease. Clinical Pulmonary Medicine, 9, 1-5. doi: 1068-0640/02/0901-001

Fernández-Fernández, F.J., Ameneiros-Lago, E., González, J., Pía, G., Martínez-Debén, F.S., \& Sesma, P. (2003). Tratamiento de la enfermedad pulmonar obstructiva crónica. Anales de Medicina Interna, 20(3), 148-155.

Foster, C., Florhaug, J.A., Franklin, J., Gottschall, L., Hrovatin, L.A., Parker, S., Doleshal, P., \& Dodge, C. (2001). A new approach to monitoring exercise training. Journal of Strength and Conditioning Research, 15(1), 109-15. doi: 10.1519/00124278-200102000-00019

García-Aymerich, J. (2005). Situación en España. En A. Agustí \& B. Celli (Ed.), Enfermedad pulmonar obstructiva crónica (pp. 19-28). Bercelona, España: Masson.

Global Initiative for Chronic Obstructive Lung Disease [GOLD]. (2016). Global strategy for diagnosis, management, and prevention of chronic obtructive pulmonary disease (updated 2016). Recuperado de http:/www.goldcopd.com/

Griffiths, T.L., Burr, M.L., Campbell, I.A., Lewia-Jenkins, V., Mullins, J., Shiels, K., (...) \& Tunbridge, J. (2000). Results at 1 year of outpatient multidisciplinary pulmonary rehabilitation: a randomised controlled trial. The Lancet, 355(9201), 362-368. doi: 10.1016/ S0140-6736(99)07042-7

Güell, M.R., Díaz, S., Rodríguez, G., Morante, F., San Miguel, M., Cejudo, P., (...) \& Servera, E. (2014). Rehabilitación respiratoria. Archivos de Bronconeumología, 50(8), 332-344. doi: 10.1016/j.arbres.2014.02.014

Harris, D.J. \& Atkinson, G. (2013). Ethical standards in sport and exercise science research: 2014 update. International Journal of Sports Medicine, 34, 1025-1028. doi: 10.1055/s-0033-1358756

Iepsen, U.W., Jorgensen, K.J., Ringbaek, T., Hansen, H., Skrubbeltrang, C., \& Lange, P. (2015). A combination of resistance and endurance training increases leg muscle strenght in COPD: An evidence-based recommendation based on systematic review with meta-analyses. Chronic Respiratory Disease, 12(2), 132-145. doi: 10.1177/1479972315575318

Karvonen, J. \& Vuorimaa, T. (1988). Heart rate and exercise intensity during sports activities: practical application. Sports Med, 5(5), 303-11. 
Lacasse, Y., Goldstein, R., Lasserson, T.J. \& Martin, S. (2006). Pulmonary rehabilitation for chronic obstructive pulmonary disease. Cochrane Database System Review, (4), CD003793. doi: 10.1002/14651858.CD003793.pub2

Mador, M.J., Bozkanat, E., Aggarwal, A., Shaffer, M., \& Kufel, T.J. (2004). Endurance and strength training in patients with COPD. Chest, 125(6), 2036-2045. doi: 10.1378/ chest.125.6.2036

Martin, D., Powers, S., Cicale, M., Collop, N., Huang, D., \& Criswell, D. (1992). Validity of pulse oximetry during exercise in elite endurance athletes. Journal of Applied Physiology, 72, 455-8.

Mayer, A.S., \& Maier, L.A. (2010). Evaluation of respiratory impairment and disability. En: R.J. Mason, V.C. Broaddus, T.R. Martin, T.E. King, D.E. Schraufnagel, J.F. Murray \& J.A. Nadel (eds.), Murray and Nadel's Textbook of Respiratory Medicine $5^{\circ}$ ed. Philadelphia, PA: Saunders Elsevier.

Menezes, A.M.B. \& Knorst, M. (2005). Situación en América Latina. En A. Agustí \& B. Celli (Ed.), Enfermedad pulmonar obstructiva crónica (pp. 29-44). Bercelona, España: Masson.

Montes, M. (2011). Bronquitis crónica en la EPOC: prevalencia e impacto sobre la enfermedad. Medicina respiratoria, 4(2), 57-64.

Nakamura, Y., Tanaka, K., Shigematsu, R., Nakagaichi, M., Inoue, M., \& Homma, T. (2008). Effects of aerobic training and recreational activities in patients with chronic obstructive pulmonary disease. International Journal of Rehabilitation Research, 31(4), 275-283. doi: 10.1097/MRR.0b013e3282fc0f81

Ortega, F., Toral, J., Cejudo, P., Villagomez, R., Sánchez, H., Castillo, J., \& Montemayor, T. (2002). Comparison of effects of strength and endurance training in patients with chronic obstructive pulmonary disease. American Journal of Respiratory and Critical Care Medicine, 166(5), 669-674. doi: 10.1164/rccm.2107081

O'Shea, S.D., Taylor, N.F., \& Paratz, J.D. (2007). Qualitative outcomes of progressive resistance exercise for people with COPD. Chronic Respiratory Disease, 4, 135-142. doi: $10.1177 / 1479972307075313$

Panton, L.B., Golden, J., Broeder, C.E., Browder, K.D., Cestaro-Seifer, D.J. \& Seifer, F.D. (2004). The effects of resistance training on functional outcomes in patients with chronic obstructive pulmonary disease. Eur J Appl Physiol, 91(4), 443-449. doi: 10.1007/s00421-003-1008-y

Puhan, M.A., Busching, G., Schunemann, H.J., VanOort, E., Zaugg, C., \& Frey, M. (2006). Interval versus continuous high-intensity exercise in chronic obstructive pulmonary disease: a randomized trial. Annals of Internal Medicine, 145, 816-825. 
Rieger, C., Garcia, F. J., Rubio, F. J., \& Marin, J. M. (2014). Classification of chronic obstructive pulmonary disease severity according to the new Global Initiative for Chronic Obstructive Lung Disease 2011 guidelines: COPD assessment test versus modified Medical Research Council scale. Archivos de Bronconeumología, 50(4), 129-134. doi: 10.1016/j.arbres.2013.09.014

Rocha Leite, M., Cipulo Ramos, E.M., Kalva-Fulho, C.A., Coelho Figueira Freire, A.P., Spolador de Alencar Silva, B., Nicolino, J., (...) \& Ramos, D. (2015). Effects of 12 weeks of aerobic training on autonomic modulation, mucociliary clearance, and aerobic parameters in patients with COPD. International Journal of COPD, 10, 2549-2557. doi: 10.2147/COPD.S81363

Saglam, M., Vardar-yagli, N., Calik-kutukcu, E., Arikan, H., Savci, S., Inal-ince, D., (...), \& Tokgozoglu, L. (2015). Functional exercise capacity, physical activity, and respiratory and peripheral muscle strength in pulmonary hypertension according to disease severity. Journal of Physical Therapy Science, 27, 1309-1312. Doi: 10.1589/jpts.271309.

Skinner, J.S. (2005). Exercise Testing and Exercise Prescription for Special Cases. Theoretical Basis and Clinical Application. $3^{\circ} \mathrm{ed}$. Lippincott: Williams \& Wilkins.

Spruit, M.A., Singh, S.J., Garvey, C., ZuWallack, R., Nici, L., Rochester, C., (...) \& ATS/ERS Task Force on Pulmonary Rehabilitation. (2013). An Official American Thoracic Society/ European Respiratory Society Key concepts and advances in pulmonary rehabilitation. American Journal of Respiratory and Critical Care Medicine, 188, 213-264. doi: 10.1164/ rccm.201309-1634ST

Troosters, T., Gosselink, R., \& Decramer, M. (2000). Short- and long-term effects of outpatient rehabilitation in patients with chronic obstructive pulmonary disease: a randomized trial. The American Journal of Medicine, 109, 207-12.

Troosters, T., Gosselink, R., Janssens, W., \& Decramer, M. (2010). Exercise training and pulmonary rehabilitation: new insights and remaining challenges. European Respiratory Review, 19, 24-29. DOI: 10.1183/09059180.00007809

Vargas, O.C. (2003). Entrenamiento físico en enfermedad respiratoria crónica. Revista ciencias de la salud, 1(2), 180-189.

Viejo, J.L. (2005). Tratamiento farmacológico. En A. Agustí \& B. Celli (Ed.), Enfermedad pulmonar obstructiva crónica (pp. 163-174). Bercelona, España: Masson.

Vonbank, K., Strasser, B., Mondrzyk, J., Marzluf, B.A., Richter, B., Losch, S., (...) \& Haber, P. (2012). Strength training increases maximum working capacity in patients with COPD - randomized clinical trial comparing three training modalities. Respiratory Medicine, 106(4), 557-563. doi: 10.1016/j.rmed.2011.11.005 
Wadell, K., Webb, K.A., Preston, M.E., Amornputtisathaporn, N., Samis, L., Patelli, J., (...) \& O'Donnell, D.E. (2013). Impact of pulmonary rehabilitation on the major dimensions of dyspnea in COPD. COPD, 10(4), 425-435. doi: 10.3109/15412555.2012.758696

Recepción: 10 de junio del 2016

Corrección: 12 de septiembre del 2016

Aceptación: 26 de octubre del 2016

Publicación: 30 de noviembre del 2016

1 Lara Blas: Graduada en Ciencias de la Actividad Física y del Deporte, Universidad del País Vasco Daniel Castillo: Licenciado en Ciencias de la Actividad Física y del Deporte, Universidad del País Vasco Oscar Lacalzada: Experto en actividad física adaptada. Centro de actividad física adaptada. Vitoria/Gasteiz. Aitor Iturricastillo: Licenciado en Ciencias de la Actividad Física y del Deporte, Universidad del País Vasco 